\title{
PSMA PET validates higher rates of metastatic disease for European Association of Urology Biochemical Recurrence Risk Groups: an international multicentre study
}

\author{
Short title: EAU risk groups and PSMA PET stage \\ Justin Ferdinandus $^{1 *}$, Wolfgang P Fendler ${ }^{1,2 *}$, Andrea Farolfi $A^{1,3}$, Samuel Washington ${ }^{4,5}$, Osama \\ Mohamad $^{6}$, Miguel H Pampaloni ${ }^{7}$, Peter JH Scott ${ }^{8}$, Melissa Rodnick ${ }^{8}$, Benjamin L Viglianti ${ }^{8}$, Matthias \\ Eiber $^{9}$, Ken Herrmann ${ }^{1,2}$, Johannes Czernin ${ }^{2}$, Wesley R Armstrong ${ }^{2}$, Jeremie Calais ${ }^{2 \dagger}$, Thomas A Hope ${ }^{7 \dagger}$, \\ Morand Piert ${ }^{8 \dagger}$ \\ ${ }^{*}$ contributed equally \\ † contributed equally
}

\begin{abstract}
Affiliations:
${ }^{1}$ Department of Nuclear Medicine, University of Duisburg-Essen and German Cancer Consortium (DKTK)-University Hospital Essen, Essen, Germany

${ }^{2}$ Ahmanson Translational Imaging Division, Department of Molecular and Medical Pharmacology, University of California Los Angeles, Los Angeles, Unites States

${ }^{3}$ Division of Nuclear Medicine, IRCCS Azienda Ospedaliero-Universitaria di Bologna, Bologna, Italy

${ }^{4}$ Department of Urology, University of California San Francisco, San Francisco, United States

${ }^{5}$ Department of Epidemiology \& Biostatistics, University of California San Francisco, San Francisco, United States

${ }^{6}$ Department of Radiation Oncology, University of California San Francisco, San Francisco, United States

${ }^{7}$ Department of Radiology and Biomedical Imaging, University of California San Francisco, San Francisco, United States

${ }^{8}$ Department of Radiology, University of Michigan, Ann Arbor, Michigan

${ }^{9}$ Department of Nuclear Medicine, Klinikum rechts der Isar, Technical University Munich, Munich, Germany
\end{abstract}

Corresponding author:

Wolfgang P. Fendler

Klinik für Nuklearmedizin, Universitätsklinikum Essen,

Hufelandstr. 55, 45147 Essen, Germany

Tel: +492017232032

Fax: +492017235964

Email: wolfgang.fendler@uk-essen.de

\author{
First author: \\ Justin Ferdinandus \\ Klinik für Nuklearmedizin, Universitätsklinikum Essen, \\ Hufelandstr. 55, 45147 Essen, Germany \\ Tel: +492017232032 \\ Fax: +49 2017235964 \\ Email: justin.ferdinandus@uk-essen.de
}


32 Keywords: EAU, risk score, prostate cancer, prostate specific membrane antigen, PSMA

33 Word count: 2228

34 Word count Abstract: 246

35 Immediate Open Access: Creative Commons Attribution 4.0 International License (CC BY) allows users

36 to share and adapt with attribution, excluding materials credited to previous publications.

37 License: https://creativecommons.org/licenses/by/4.0/.

38 Details: https://jnm.snmjournals.org/page/permissions.

(c) 


\section{ABSTRACT}

The European Association of Urology (EAU) Prostate Cancer Guidelines Panel recommends risk groups for biochemically recurrent prostate cancer $(B C R)$ to identify men at high risk of progression or metastatic disease. The rapidly growing availability of PSMA-directed PET imaging (PSMA PET) will impact prostate cancer staging. We determined the rates of local and metastatic disease in recurrent and persistent prostate cancer stratified by EAU BCR risk groups and biochemical persistence (BCP). Methods: Patients with $\mathrm{BCR} / \mathrm{BCP}$ were enrolled under the same prospective clinical trial protocol conducted at three sites ( $n=1777,91 \%$; UCLA $n=662$, NCT02940262; UCSF $n=508$, NCT03353740; Michigan, $\mathrm{n}=607$, NCT03396874); 183 patients with BCP from Universities of Essen, Bologna, and Munich were included retrospectively. Patients with $B C R$ had to have sufficient data to determine EAU risk score. Multivariate, binomial logistic regression models were applied to assess independent predictors of $\mathrm{M} 1$ disease. Results: A total of 1960 patients were included. Post-RP EAU BCR low risk, EAU BCR high risk, and BCP groups yield distant metastatic (M1) detection in 43/176 (24\%), 342/931 (37\%), and 154/386 (40\%) of patients. For post-radiotherapy EAU BCR low risk and EAU BCR high risk groups, $\mathrm{M} 1$ detection rate was 113/309 (37\%) and 110/158 (70\%), respectively. $B C P$, high risk $B C R$ and higher levels of serum PSA were significantly associated with PSMA PET M1 disease in multivariate regression analysis. PSMA-PET revealed no disease in $25 \%$ and locoregional only disease in $33 \%$ of patients with post-RP or postradiotherapy EAU BCR high risk. Conclusion: Our findings support the new EAU classification; EAU BCR high-risk groups have higher rates of metastatic disease on PSMA PET than the low-risk groups. Discordant subgroups, including metastatic disease in low risk and no disease in high risk patients warrant inclusion of PSMA PET stage to refine risk assessment. 


\section{INTRODUCTION}

After primary curative-intent treatment for prostate cancer $(\mathrm{PCa})$ with radical prostatectomy (RP) or radiotherapy, approximately one out of four men experience biochemical recurrence (BCR) (1).

The incidence and outcomes of BCR are variable. A novel European Association of Urology (EAU) risk-scoring system combines PSA doubling time (PSA-DT), gleason score (GS), and interval from primary therapy to biochemical failure (IBF), to identify patients at high risk for metastases and early disease progression (2). Of note, PSA persistence (BCP), was described as a different pattern of relapse, which is associated with worse oncological outcomes and was therefore not stratified into risk groups (3).

Tilki et al. validated the EAU BCR risk score using survival data from an extensive dataset of post-RP patients from their centre. Metastatic progression-free and overall survival were significantly different; However, the prognostic accuracy for metastasis-free survival (c-index 0.67 ) or disease specific survival (c-index 0.69 ) was moderate, warranting further refinement of this classification (4).

PSMA-targeted positron emission tomography (PSMA PET) has demonstrated high detection rates and accuracy for the localization of prostate cancer metastases (5). The improved accuracy of PSMA PET along with impact on management led to its inclusion in the EAU guidelines as well as FDA approval for imaging primary and recurrent

disease $(6,7)$. Several trials evaluating the potential of PSMA PET guided therapy to achieve improved outcome are currently underway or recently published $(8,9)$. PSMA PET disease extent was associated with time to progression in patient candidates for salvage radiotherapy and may thus offer independent prognostic value at BCR and BCP (10). 
The aim of this study was to assess disease extent in patients with EAU BCR high risk, low risk, and BCP using PSMA PET to identify subgroups of undetectable (TONOMO), locoregional (Tr/N1), or distant metastatic (M1) disease.

\section{METHODS}

This is a multicentric, single-arm analysis of Patients with recurrent or persistent PSA after curative treatment of prostate cancer. Biochemical recurrence was defined as a PSA of 0.2 or more $\mathrm{ng} / \mathrm{mL}$ measured more than 6 weeks after prostatectomy or a PSA of 2 or more $\mathrm{ng} / \mathrm{mL}$ rise above nadir following radiation therapy. Biochemical persistence was defined as PSA nadir $>0.1 \mathrm{ng} / \mathrm{ml}$ within 12 weeks after RP. The final database consisted of 1960 patients with either BCR $(n=1574)$ or BCP $(n=386)$. The majority of patients were enrolled under the same prospective clinical trial protocol conducted at three sites $(n=1777,91 \% ;$ UCLA $n=662$, NCT02940262; UCSF $n=508$, NCT03353740; Michigan, n=607, NCT03396874); 183 patients with BCP from Universities of Essen, Bologna, and Munich were included retrospectively. In total 587/1960 (30\%) patients have been reported previously $(5,6,11)$. The study was approved by institutional review boards at each site.

Patients were eligible if they had a history of histopathology-proven prostate adenocarcinoma and BCR or BCP after curative-intent radiotherapy or prostatectomy. Further, BCR patients had to have sufficient data to determine risk group: PSA-DT and GS for recurrence after post-prostatectomy, IBF and GS post-radiotherapy. Patients had to have complete reading data. Patients with known metastases prior to PSMA PET, prior salvage treatment or PSMA PET within three months after curative treatment were not eligible for this analysis. A flow chart for patient inclusion is shown in Figure 1. 
Detailed imaging procedures were reported previously (5) and scans were acquired in accordance with the international guideline (12). In brief, whole-body PET was acquired from skull to mid thighs. PET was performed as hybrid imaging with CT or MRI based on availability and contraindications. For PET/CT, a diagnostic contrast-enhanced CT was obtained before the PET scan. For PET/MRI, an abbreviated pelvis PET/MRI was obtained following a whole-body protocol after the PET scan. PSMA PET findings were interpreted using Prostate Cancer Molecular Imaging Standardized Evaluation (PROMISE) criteria (13).

Descriptive statistics were used to report patient characteristics and disease extent. Multivariate, binomial logistic regression models were applied to assess independent predictors of M1 disease. Analyses were performed using R v.3.4.0 (R Foundation for Statistical Computing, Vienna, Austria). Figure parts were created using BioRender Software.

\section{RESULTS}

Table 1 lists patient characteristics and PSMA PET stage. Median PSA serum level at time of PSMA PET was $1.76 \mathrm{ng} / \mathrm{mL}$ (IQR 4.28). PSA values differed post-RP (Median $1.0 \mathrm{ng} / \mathrm{mL}, \mathrm{IQR} 2.4$ ) vs. post-radiotherapy (Median $5.1 \mathrm{ng} / \mathrm{mL}, \mathrm{IQR} 6.4$ ). A total of 1493 (76\%) patients received primary RP and 467 (24\%) patients received primary radiotherapy. More than $60 \%$ of patients in the post-RP group had PSA $<2.0 \mathrm{ng} / \mathrm{mL}$, whereas - also due to different BCR definition - majority of post-radiotherapy patients had $P S A \geq 2 \mathrm{ng} / \mathrm{mL}$. Median [IQR] time since initial therapy was longest in the respective EAU low risk groups (post-RP, 9.6 [7.4] months; post-radiotherapy, 7.4 [6.9] months). PSMA 
PET localized disease in 1515/1960 (77\%) patients. Figure 2A shows PSMA PET detected disease extent separate for EAU BCR risk groups and BCP.

PSMA PET revealed M1 disease within the post-RP group in 43/176 (24\%), 342/931 (37\%), and 154/386 (40\%) of EAU BCR low risk, high risk, and BCP patients, respectively. Within the post-radiotherapy group, M1 disease was detected in 113/309 (37\%) and 110/158 (70\%) of EAU BCR low and high risk patients, respectively. Bone metastases were detected in 19/176 (11\%), 201/931 (37\%), 88/386 (23\%) for post-RP EAU BCR low risk, high risk and BCP subgroups, and in 16/309 (5\%) and 15/158 (10\%) for post-radiotherapy EAU BCR low and high risk subgroups, respectively. Visceral metastases were detected in 3 to $6 \%$ for post-RP subgroups, and $16 / 309(5 \%)$ as well as 15/158 (10\%) for post-radiotherapy EAU BCR low and high risk subgroups, respectively. The number of involved regions differed among the different risk groups. Three or more involved metastatic regions were detected in 38/176 (22\%), 287/931 (31\%), 110/386 (29\%) of post-RP EAU BCR low risk, high risk and BCP patients, as well as 102/309 (33\%) and 92/158 (58\%) of post-radiotherapy EAU BCR low and high risk patients, respectively.

PSMA PET revealed no disease in 58/176 (33\%), 275/931 (30\%), and 85/386 (22\%) of post RP EAU low risk, high risk and BCP subgroups. Post-radiotherapy subgroups were PET negative in 20/309 (7\%) low risk patients and 7/158 (4\%) EAU BCR high risk, respectively.

Figure $2 \mathrm{~B}$ shows a Forest plot for odds ratios $(\mathrm{OR})$ derived from multivariate regression. Higher PSA levels, EAU BCR high risk (OR 2.91, 95\% $\mathrm{Cl} 2.18-3.93$ ), and $\mathrm{BCP}$ (OR 3.08, 95\% $\mathrm{Cl}$ 2.12-4.48) were significantly associated with PSMA PET M1 disease, whereas type of initial therapy was not. 


\section{DISCUSSION}

${ }^{68}$ Ga-PSMA-11 and ${ }^{18}$ F-DCFPyL PET were recently approved by FDA based on high accuracy for prostate cancer staging $(5,11,14)$. Approval of PSMA-ligand PET is expected to enable broad availability for staging BCR or BCP in the near future. Our findings present a detailed map of disease extent in the EAU BCR risk groups or BCP. Observed intra- and inter-group heterogeneities for PET stage come with important implications for the EAU classification system.

At first, PSMA PET stratified EAU or BCP groups into relevant subgroups with undetectable, locoregional, or distant metastatic disease. After RP, about one third of patients stratified into each of these three subgroups, with somewhat higher rates for metastatic disease in the BCR high risk or BCP patients. We present a single time point assessment; however, PET stage was associated with time to progression in a previous prospective study on BCR (10).

Recently, Dong et al. noted in a pooled analysis of 145 patients following prostatectomy or radiotherapy, that EAU BCR high risk group was associated with a higher PSMA-PET positivity rate (15). In this study, we assessed patients following prostatectomy or radiotherapy separately, and found similar rates for PET positivity, however higher rates for metastatic disease in patients with EAU high as compared to low risk. In addition, PSMA PET identified subgroups with discordant findings for EAU risk label versus PET stage: $30 \%$ post-RP EAU high risk patients had undetectable disease whereas $24 \%$ low risk patients had metastatic disease, including $11 \%$ bone and $6 \%$ visceral metastases. Discordant findings together with previous evidence by Emmett et al indicate additional prognostic value of PSMA PET to be considered for future risk assessment. 
Second, disease extent detected by PSMA PET was higher in post-radiotherapy versus RP patients: Post-radiotherapy EAU low risk patients yield PSMA PET M1 rates similar to post-RP high risk or BCP. Strikingly, more than two thirds of post-radiotherapy high risk patients had metastases, including bone metastases in $31.4 \%$ and visceral metastases in $14.5 \%$. In patients with EAU high risk, the incidence of M1 postradiotherapy was nearly twice that of post-RP; the rate of $\mathrm{M} 1$ visceral disease was more than two times higher. Due to different BCR definitions, this can be attributed to higher PSA values post-radiotherapy (Median $5.1 \mathrm{ng} / \mathrm{mL}$, IQR 6.4) compared to the post-RP group (Median $1.0 \mathrm{ng} / \mathrm{mL}, \mathrm{IQR}$ 2.4). Accordingly, initial therapy was not a significant predictor of metastatic disease in multivariate regression analysis with PSA levels included. We assume that heterogeneous PSMA PET disease extent reflect clinical reality, i.e. post-radiotherapy or -RP BCR risk groups will likely present different outcomes despite sharing the same risk label. To account for these differences, PSA level as well as RP/radiotherapy specific risk group nomenclature should be considered for risk assessment. We confirm previously reported association of PSA with PSMA PET M1 disease. PSA level was a stronger predictor of the presence of M1 disease than EAU risk groups. BCR or BCP states are defined using PSA kinetics without specific inclusion of individual PSA values. However, in the transition phase with limited availability of PSMA PET, PSA level will help identify patients at high risk who may benefit from PSMA PET staging. 


\section{CONCLUSION}

In summary, we demonstrate that men with high risk BCR according to EAU Prostate Cancer Guidelines Panel and BCP have higher rates of metastatic disease. Discordant subgroups, including metastatic disease in low risk and no disease in high risk patients warrant inclusion of PSMA PET stage to refine risk assessment.

\section{DISCLOSURE}

JF has received fees from Eisai outside of the submitted work. WPF reports fees from Calyx (consultant), RadioMedix (image review), Bayer (speakers bureau), and Parexel (image review) outside of the submitted work. ME reports personal fees from Blue Earth Diagnostics, Progenics Pharmaceuticals, Amgen, Parexel, Bayer and Point Biopharma, research support from Blue Earth Diagnostics and a patent application for rhPSMA outside of the submitted work. KH reports personal fees from Bayer, personal fees and other from Sofie Biosciences, personal fees from SIRTEX, non-financial support from $A B X$, personal fees from Adacap, personal fees from Curium, personal fees from Endocyte, grants and personal fees from BTG, personal fees from IPSEN, personal fees from Siemens Healthineers, personal fees from GE Healthcare, personal fees from Amgen, personal fees from Novartis, personal fees from ymabs, outside the submitted work. JC is a cofounder and holds equity in Sofie Biosciences and Trethera Therapeutics. Intellectual property patented by the University of California has been licensed to Sofie Biosciences and Trethera Therapeutics. This intellectual property was not used in the current study. JC is consultant for Blue Earth Diagnostics, Progenics Radiopharmaceuticals, and Radiomedix, outside the submitted work. TAH is on a consultancy or advisory board for Curium and Ipsen; performs research for Clovis 
228 Oncology and Philips; and is a trial participant for Novartis and AAA. All others have 229 nothing to disclose.

\section{KEY POINTS}

233 QUESTION: Does the new EAU Risk classification identify distinct patterns of disease 234 spread in PSMA PET?

235 PERTINENT FINDINGS: In this multicentre, international study, including 1960 men with

236 biochemically recurrent $(B C R)$ or persistent $(B C P)$ prostate cancer, we found that $E A U$ 237 high risk $\mathrm{BCR}$ and $\mathrm{BCP}$ was significantly associated with a higher risk of metastatic 238 disease in PSMA PET. However, PSMA PET also found patients with discordant patterns, 239 i.e. no detected disease in high risk patients and metastatic disease in low risk patients. 240 IMPLICATIONS FOR PATIENT CARE: PSMA PET validates the novel EAU BCR risk 241 classification. In addition, it may further refine risk assessment in this cohort. 


\section{REFERENCES}

1. Pompe RS, Gild P, Karakiewicz PI, et al. Long-term cancer control outcomes in patients with biochemical recurrence and the impact of time from radical prostatectomy to biochemical recurrence. Prostate. 2018;78:676-681.

2. Van den Broeck $T$, van den Bergh RCN, Briers E, et al. Biochemical Recurrence in Prostate Cancer: the european Association of Urology Prostate Cancer Guidelines Panel Recommendations. Eur Urol Focus. 2020;6:231-234.

3. Fossati N, Karnes RJ, Colicchia M, et al. Impact of early Salvage Radiation Therapy in Patients with persistently elevated or rising prostate-specific Antigen after radical Prostatectomy. Eur Urol. 2018;73:436-444.

4. Tilki D, Preisser F, Graefen M, Huland H, Pompe RS. External Validation of the european Association of Urology biochemical Recurrence Risk Groups to predict Metastasis and Mortality after radical Prostatectomy in a european Cohort. Eur Urol. 2019;75:896-900.

5. Fendler WP, Calais J, Eiber M, et al. Assessment of 68Ga-PSMA-11 PET Accuracy in Localizing recurrent Prostate Cancer: a prospective single-arm clinical Trial. JAMA Oncol. 2019;5:856-863.

6. Fendler WP, Ferdinandus J, Czernin J, et al. Impact of (68)Ga-PSMA-11 PET on the Management of recurrent Prostate Cancer in a prospective single-arm clinical Trial. $J$ Nucl Med. 2020;61:1793-1799.

7. Sartor O, Hope TA, Calais J, Fendler WP. Oliver Sartor talks with Thomas A. Hope, Jeremie Calais, and Wolfgang P. Fendler about FDA Approval of PSMA. J Nucl Med. 2021;62:146-148.

8. Calais J, Armstrong WR, Kishan AU, et al. Update from PSMA-SRT Trial NCT03582774: a randomized Phase 3 Imaging Trial of prostate-specific Membrane Antigen Positron Emission Tomography for Salvage Radiation Therapy for Prostate Cancer Recurrence powered for clinical Outcome. Eur Urol Focus. 2020;7:238-240.

9. Phillips R, Shi WY, Deek M, et al. Outcomes of Observation vs stereotactic ablative Radiation for oligometastatic Prostate Cancer: The ORIOLE Phase 2 randomized clinical Trial. JAMA Oncol. 2020;6:650-659. 
10. Emmett L, Tang R, Nandurkar R, et al. 3-Year Freedom from Progression after (68)Ga-PSMA PET/CT-triaged Management in Men with biochemical Recurrence after radical Prostatectomy: Results of a prospective multicenter Trial. J Nucl Med. 2020;61:866-872.

11. Farolfi A, Gafita A, Calais J, et al. (68)Ga-PSMA-11 Positron Emission Tomography detects residual Prostate Cancer after Prostatectomy in a multicenter retrospective Study. J Urol. 2019;202:1174-1181.

12. Fendler WP, Eiber M, Beheshti M, et al. (68)Ga-PSMA PET/CT: Joint EANM and SNMMI procedure guideline for prostate cancer imaging: version 1.0. Eur J Nucl Med Mol Imaging. 2017;44:1014-1024.

13. Eiber M, Herrmann $\mathrm{K}$, Calais J, et al. Prostate Cancer molecular Imaging standardized Evaluation (PROMISE): proposed miTNM Classification for the Interpretation of PSMA-Ligand PET/CT. J Nucl Med. 2018;59:469-478.

14. Pienta KJ, Gorin MA, Rowe SP, et al. A Phase $2 / 3$ prospective multicenter Study of the diagnostic Accuracy of Prostate specific Membrane Antigen PET/CT with (18)FDCFPyL in Prostate Cancer Patients (OSPREY). J Urol. 2021;206:52-61.

15. Dong L, Su Y, Zhu Y, et al. The european Association of Urology biochemical Recurrence Risk Groups predict Findings on PSMA PET in Patients with biochemically recurrent Prostate Cancer after radical Prostatectomy. J Nucl Med. 2021; In press. 
Table 1: Patient characteristics and PSMA PET stages

\begin{tabular}{|c|c|c|c|c|c|}
\hline & \multicolumn{3}{|c|}{ Prostatectomy } & \multicolumn{2}{|c|}{ Radiotherapy } \\
\hline & $\begin{array}{c}\text { EAU } \\
\text { low risk } \\
(n=176)\end{array}$ & $\begin{array}{c}\text { EAU } \\
\text { high risk } \\
(n=931)\end{array}$ & $\begin{array}{l}\text { Biochemical Persistence } \\
\qquad(n=386)\end{array}$ & $\begin{array}{c}\text { EAU } \\
\text { low risk } \\
(n=309)\end{array}$ & $\begin{array}{c}\text { EAU } \\
\text { high risk } \\
(n=158)\end{array}$ \\
\hline \multicolumn{6}{|l|}{ Age } \\
\hline Median [IQR] & $71[9.3]$ & $69[9.1]$ & 70 [12] & $73[9.6]$ & $72[9.1]$ \\
\hline \multicolumn{6}{|l|}{ PSA } \\
\hline$<0.5 \mathrm{ng} / \mathrm{ml}$ & $60(34.1 \%)$ & $302(32.4 \%)$ & 69 (17.9\%) & $3(1.0 \%)$ & $2(1.3 \%)$ \\
\hline$\geq 0.5$ to $<1.0 \mathrm{ng} / \mathrm{ml}$ & $38(21.6 \%)$ & $178(19.1 \%)$ & $175(45.3 \%)$ & $4(1.3 \%)$ & $4(2.5 \%)$ \\
\hline$\geq 1.0$ to $<2.0 \mathrm{ng} / \mathrm{ml}$ & $20(11.4 \%)$ & $174(18.7 \%)$ & $43(11.1 \%)$ & $7(2.3 \%)$ & $5(3.2 \%)$ \\
\hline$\geq 2.0$ to $<5.0 \mathrm{ng} / \mathrm{ml}$ & $34(19.3 \%)$ & $159(17.1 \%)$ & $41(10.6 \%)$ & $134(43.4 \%)$ & $62(39.2 \%)$ \\
\hline$\geq 5.0 \mathrm{ng} / \mathrm{ml}$ & $24(13.6 \%)$ & $118(12.7 \%)$ & $58(15.0 \%)$ & $161(52.1 \%)$ & $85(53.8 \%)$ \\
\hline \multicolumn{6}{|l|}{ PSADT (months) } \\
\hline Median [IQR] & 20 [18] & $4.2[5.2]$ & $4.5[5.8]$ & $8.5[11]$ & $4.1[5.7]$ \\
\hline \multicolumn{6}{|l|}{ Gleason Score } \\
\hline 6 & $30(17.0 \%)$ & $42(4.5 \%)$ & $17(4.4 \%)$ & 97 (31.4\%) & $2(1.3 \%)$ \\
\hline 7 & $146(83.0 \%)$ & $507(54.5 \%)$ & $168(43.5 \%)$ & $212(68.6 \%)$ & $27(17.1 \%)$ \\
\hline 8 & - & $168(18.0 \%)$ & $79(20.5 \%)$ & - & $55(34.8 \%)$ \\
\hline $9-10$ & - & $214(23.0 \%)$ & $122(31.6 \%)$ & - & $74(46.8 \%)$ \\
\hline \multicolumn{6}{|l|}{ IBF (months) } \\
\hline Median [IQR] & 83 [78] & $44[51]$ & 34 [55] & 88 [84] & $41[65]$ \\
\hline \multicolumn{6}{|l|}{ Adjuvant RT after RP } \\
\hline Adjuvant RT & $50(28.4 \%)$ & $368(39.5 \%)$ & $78(20.2 \%)$ & - & - \\
\hline No adjuvant RT & $126(71.6 \%)$ & $563(60.5 \%)$ & $308(79.8 \%)$ & $309(100 \%)$ & $158(100 \%)$ \\
\hline \multicolumn{6}{|l|}{ PSMA PET stage } \\
\hline TONOMO (no disease) & $58(33.0 \%)$ & $275(29.5 \%)$ & $85(22.0 \%)$ & $20(6.5 \%)$ & $7(4.4 \%)$ \\
\hline Tr/N1 M0 (locoregional) & 75 (42.6\%) & $314(33.7 \%)$ & $147(38.1 \%)$ & $176(57.0 \%)$ & 41 (25.9\%) \\
\hline Any M1 (metastatic) & $43(24.4 \%)$ & $342(36.7 \%)$ & $154(39.9 \%)$ & $113(36.6 \%)$ & $110(69.6 \%$ \\
\hline \multicolumn{6}{|l|}{ M1 group } \\
\hline M1a only & $13(7.4 \%)$ & $102(11.0 \%)$ & $53(13.7 \%)$ & 49 (15.9\%) & $30(19.0 \%)$ \\
\hline Any $M 1 b^{*}$ & $19(10.8 \%)$ & $201(21.6 \%)$ & $88(22.8 \%)$ & $48(15.5 \%)$ & $65(41.1 \%)$ \\
\hline Any M1c & $11(6.2 \%)$ & $39(4.2 \%)$ & $13(3.4 \%)$ & $16(5.2 \%)$ & $15(9.5 \%)$ \\
\hline \multicolumn{6}{|l|}{ No. M1 regions } \\
\hline 0 & $133(75.6 \%)$ & $589(63.3 \%)$ & $232(60.1 \%)$ & $196(63.4 \%)$ & $48(30.4 \%)$ \\
\hline $1-2$ & $5(2.8 \%)$ & $55(5.9 \%)$ & $44(11.4 \%)$ & $11(3.6 \%)$ & $18(11.4 \%)$ \\
\hline$\geq 3$ & $38(21.6 \%)$ & $287(30.8 \%)$ & $110(28.5 \%)$ & $102(33.0 \%)$ & $92(58.2 \%)$ \\
\hline
\end{tabular}

$\mathrm{PSA}=$ prostate specific antigen, IQR = inter quartile range, PSMA stages according to PROMISE criteria $(13),{ }^{*}=$ not including M1c 
Figure 1: Study flow chart

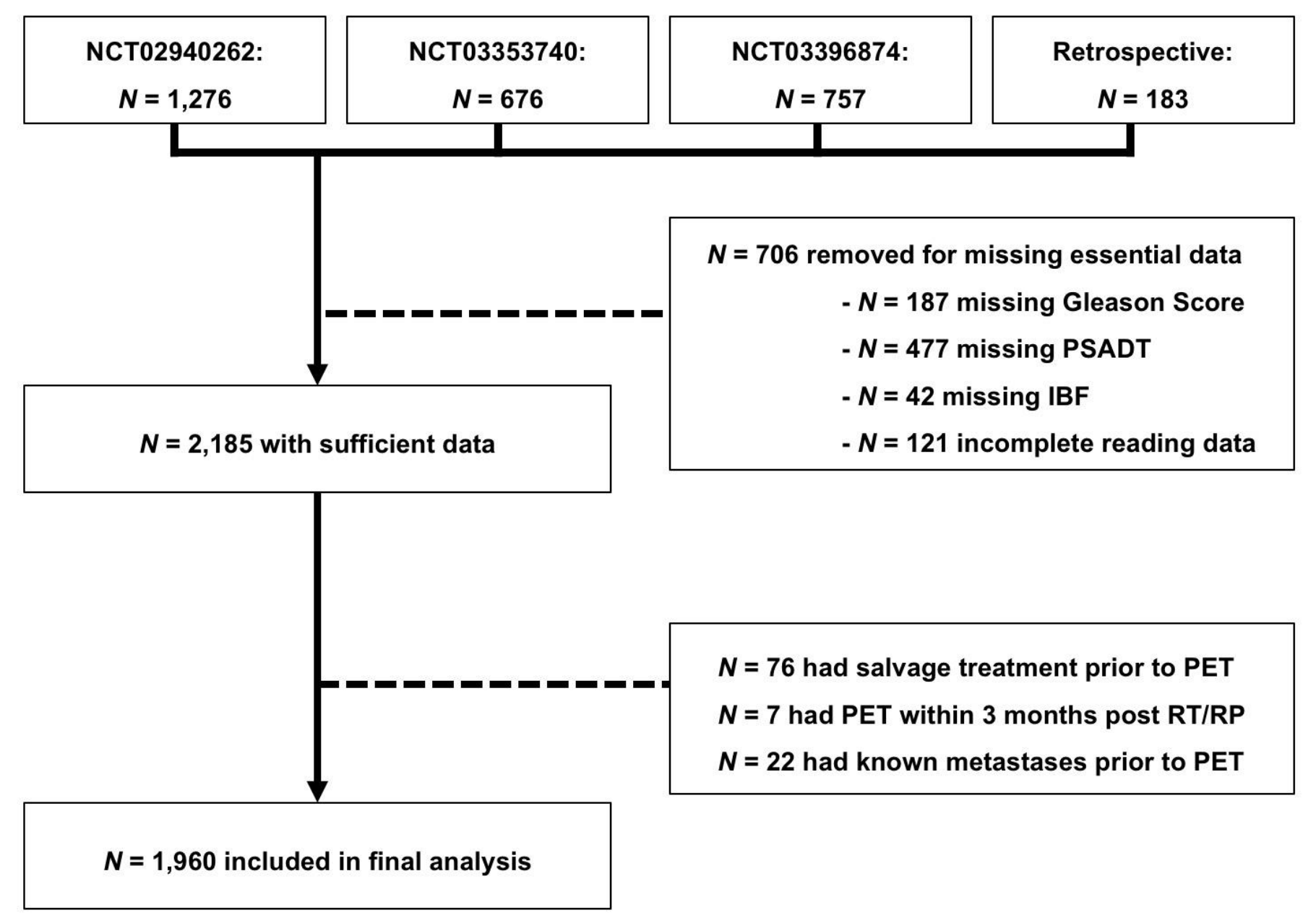


Figure 2: PET disease extent in EAU BCR low risk, high risk and in BCP patients (A) and predictors of PET M1 disease (B)

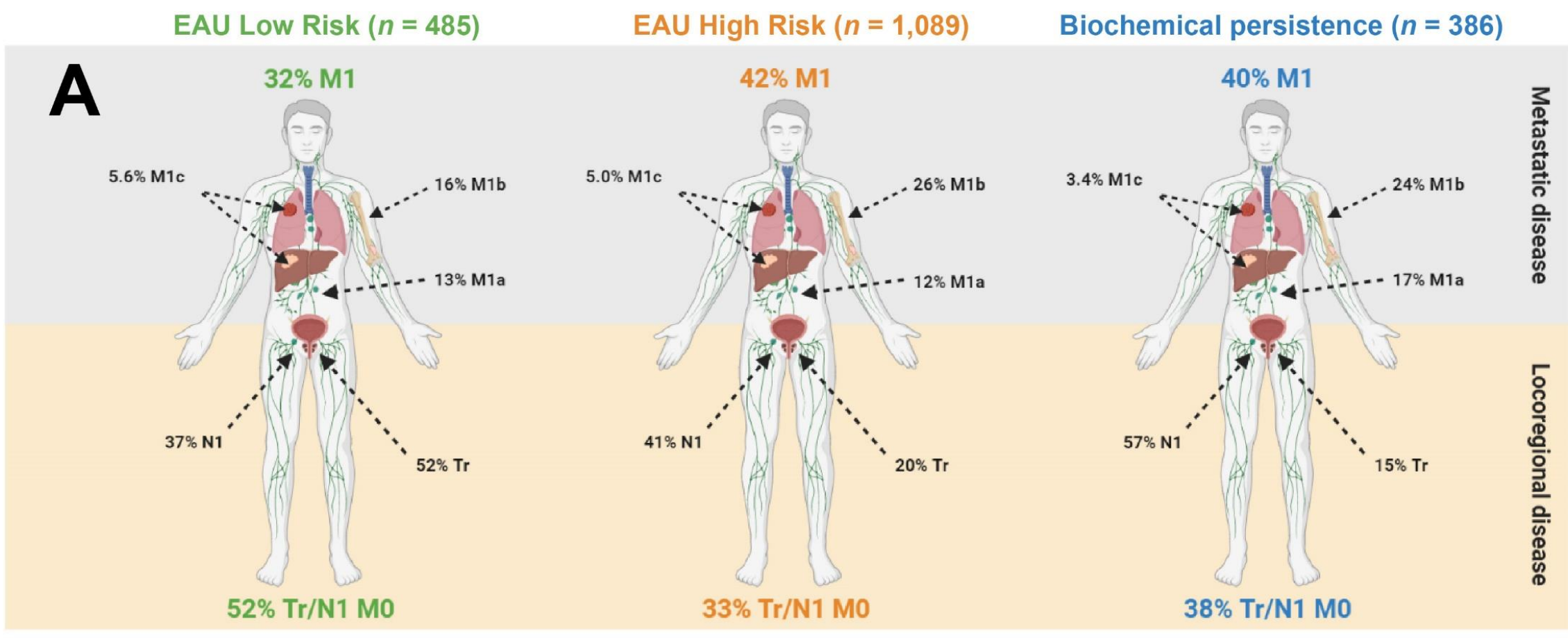

Category
Initial therapy

PSA

$\begin{array}{rr}\text { group } & \text { no. M0 (\%) } \\ \text { prostatectomy } & 954(79.6) \\ \text { radiotherapy } & 244(20.4) \\ <0.5 \mathrm{ng} / \mathrm{ml} & 366(30.6) \\ \geq 0.5 \text { to }<1.0 \mathrm{ng} / \mathrm{ml} & 276(23.0) \\ \geq 1.0 \text { to }<2.0 \mathrm{ng} / \mathrm{ml} & 154(12.9) \\ \geq 2.0 \text { to }<5.0 \mathrm{ng} / \mathrm{ml} & 236(19.7) \\ \geq 5.0 \mathrm{ng} / \mathrm{ml} & 166(13.9) \\ \text { EAU low risk } & 329(27.5) \\ \text { EAU high risk } & 637(53.2) \\ \text { Biochemical Persistence } & 232(19.4)\end{array}$

no. M1 (\%) $539(70.7)$$$
70(9.2)
$$
$123(16.1)$ $95(12.5)$ $194(25.5)$ $280(36.7)$

$156(20.5)$

154 (20.2)

\begin{tabular}{|c|c|}
\hline OR (Cl95) & $P$ \\
\hline & Reference \\
\hline $1.08(0.79-1.49)$ & ) $P=0.612$ \\
\hline & Reference \\
\hline $2.24(1.59-3.17)$ & ) $P<0.001$ \\
\hline $3.17(2.21-4.58)$ & ) $P<0.001$ \\
\hline $5.56(3.96-7.87)$ & ) $P<0.001$ \\
\hline $.16(8.52-17.55)$ & ) $P<0.001$ \\
\hline & Reference \\
\hline $2.91(2.18-3.93)$ & ) $P<0.001$ \\
\hline $3.08(2.12-4.48)$ & ) $P<0.00$ \\
\hline
\end{tabular}

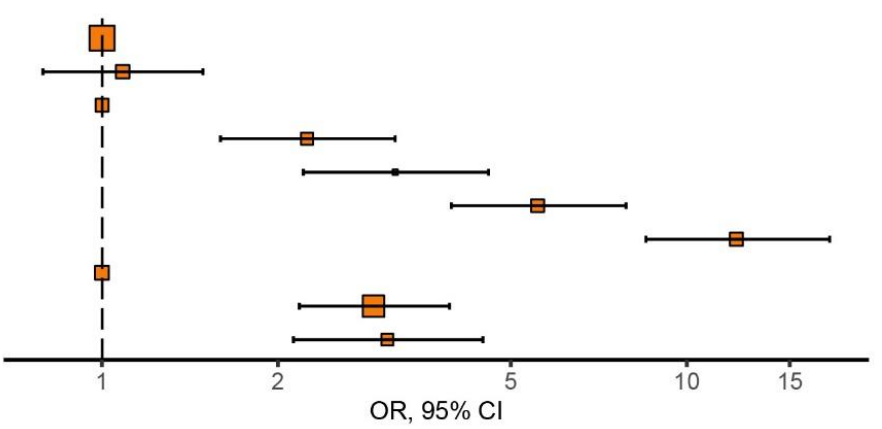




\section{Graphical Abstract}

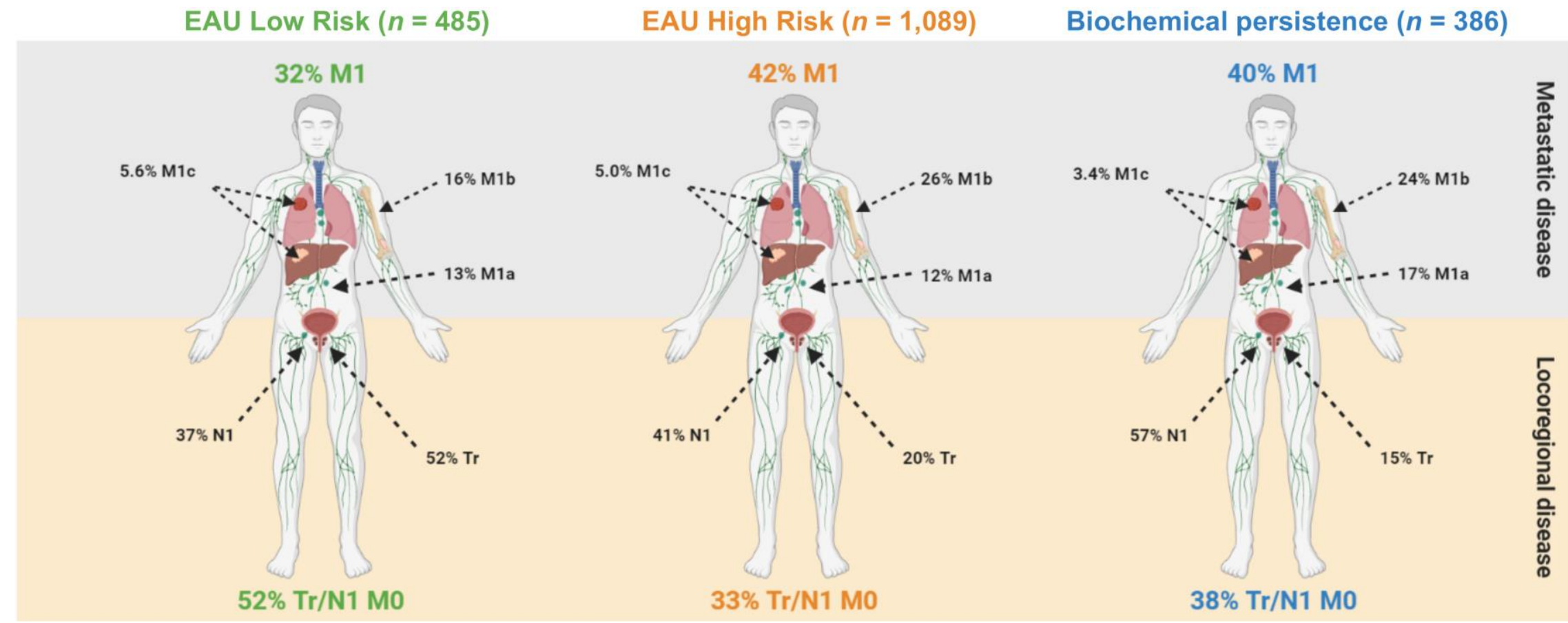

